\title{
Design and Implementation of an Around View Monitoring System of Selecting Scope of View
}

\author{
Sweung-Hwan Cheon ${ }^{1 *}$, Jong-Wook Jang ${ }^{2}$ and Si-Woong Jang ${ }^{3}$ \\ ${ }^{1}$ Department of IT Convergence, DONG-EUI University, 614-701, Korea \\ ${ }^{2}$ Department of Computer Engineering, DONG-EUI University, 614-701, Korea \\ ${ }^{3}$ Department of Computer Science, DONG-EUI University, 614-701, Korea \\ 13764@deu.ac.kr,jwjang@deu.ac.kr,swjang@deu.ac.kr
}

\begin{abstract}
Recently certain driver convenience systems including ADAS (Advanced Driver Assistance System) such as navigation and GPS, and driver's infotainment devices such as DVD players have been produced. AVM systems of these driver convenience systems support safety driving by providing situations around the vehicle to a driver. A driver can't fully monitor the situation around the vehicle at a glance using only rearview mirrors, a room mirror, and front and rear cameras, and using of only these devices causes blind spot which hinders driver's safety. Blind spot is cause of personal injury and vehicle damage when parking vehicle, passing on a narrow street and turning left or right.

Therefore studies on AVM systems have actively been progressing which provides surroundings of vehicles for drivers. Though existing AVM systems combines video images from four cameras and provides a driver with a full vision around the vehicle with no blind spots, the system has limitations that its monitoring area is narrow and fixed. Thus they are suitable for parking but are not suitable for monitoring situations around vehicle in various purposes. Therefore, in this paper, we will design and implement AVM system with adjustable around view to be shown that the proposed system provides multiple scopes of around views.
\end{abstract}

Keywords: Around View Monitoring System, camera, corners detection

\section{Introduction}

Upon the enormous improvement over the automobile industry, many driving auxiliary systems for drivers' convenience, such as an auto parking system or an AVM system have been developed [1,6]. An AVM system is a system equipped with several cameras which provides a driver with the surroundings of a car. An AVM system provides an around view image through the display of a driver, going through the image process consisting of radial distortion correction, perspective distortion correction and image synthesis [2]. The current AVM systems have weak points that human intervention is required at the stage of perspective distortion correction among the foregoing stages and that a synthesized image is provided only with a fixed scope of view. In order to solve such points, straight lines and corners should be detected automatically and a driver should be able to select his desired scope of view to see a synthesized image.

This paper designs and implements an AVM system which provides methods to detect straight lines and corners automatically and enables a driver to see a synthesized image from the scope of view that he wants, so that such an AVM system may be easily applied to a car.

In Chapter 2 of this paper, studies related to currently released AVM systems, etc. will be explained, in Chapter 3, design of AVM system to detect straight lines and corners and select scope of view, in Chapter 4, implementation of the proposed methods and in Chapter 5, conclusion. 


\section{Related Studies}

An around view monitoring system is a driving auxiliary system to assist a driver driving and parking a car by way of showing an around view image after receiving images from the respective cameras set on the front, rear, left and right sides of the car and correcting and synthesizing such images. AVM system manufactures include Hyundai Mobis in Korea, Nissan in Japan, BMW in Germany, etc. Such manufacturers have different technologies to correct images and each of their AVM systems has strong and weak points.

The AVM system of Hyundai Mobis provides a driver with an around view image through a display after synthesizing images from a wide angle (185 degrees) cameras set on the front, rear, left and right sides of a car as one image. However, such a system is installed only in high class cars and the cost for its option is also expensive, amounting to approximately KRW 1 million.

The Around View Monitor System of Nissan shows surroundings of a car, in real time, through a 7-inch color screen by means of images inputted by four wide cameras (180 degrees) installed on the front, rear, left and right sides of a car. This system has a weak point that it operates only when a car runs at low speed below $10 \mathrm{Km}$ per hour [3].

The Surround View of BMW has a function to show in advance the trace to turn the handle by showing a synthesized image from the left, right and rear views, except for the front. However, since there is no image from the front side, drivers inexperienced in driving may feel uncomfortable [4].

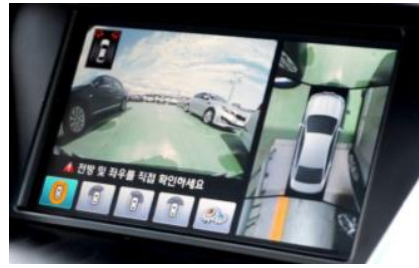

(a) Hyundai AVM System

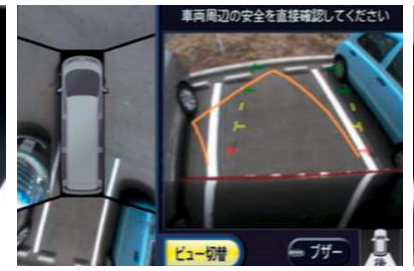

(b) Nissan AVM System

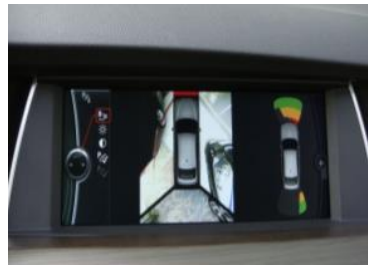

(c) BMW Surround-View

Figure 1. Existing AVM Systems

Those systems provide images so as to enable drivers to recognize the surroundings of their cars by use of cameras. However, there is a problem: since the scope of view is fixed, the driver cannot select and see the field that he/she wants.

This paper proposes an AVM system selecting scope of view so that a driver may select the scope of view that he/she wants and see the around view image. The AVM system selecting scope of view provides a driver with wider scope of view as well as the surroundings of his car through an image synthesis in real time. Moreover, the AVM system implemented in this paper has a strong point to minimize human intervention by means of auto-detection of straight lines and corners.

\section{Design of an AVM System Selecting Scope of View}

In order to implement an AVM system selecting scope of view, it is firstly necessary to obtain corrected distortion images and to automatically detect straight lines and corners on the inside of the grid patterns of such images. In addition, it is needed to make virtual straight lines and corners on the outside of the grid patterns. An AVM system which synthesizes images by use of the foresaid two methods is designed.

An AVM system uses wide angle cameras of 180 degrees or more. The character of a wide angle camera leads to radial distortion. The phenomenon of radial distortion refers to the phenomenon that a point of an image is not located on a normal place but on the inside or outside of the normal place in the radial direction from the lens. Figure 2 shows 
a sphere-based method to correct distorted images which is used in this paper, among various methods to correct such distortion [5]

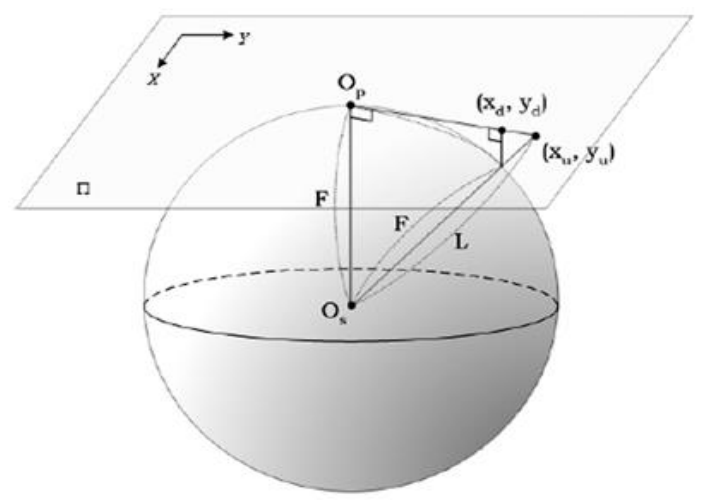

Figure 2. Correcting Distorted Image based on Sphere

The design includes auto detection of straight lines and corners after obtaining corrected distortion images by use of the sphere-based method to correct distorted images.

First of all, edges are detected and the border lines of corrected distortion images are extracted. Among various detectors such as Homogeneity Operator, Difference Operator, Edge Image Thresholding, etc., this paper adopts Homogeneity Operator, the simplest and quickest edge detector. The Homogeneity Operator Edge Detector uses a method to reduce 8 pixels from the center pixel of a $3 \times 3$ sized image, respectively and the biggest value is displayed among the absolute values of the respective differences.

Next, all the straight elements in the detected edge image should be extracted. In this paper, the Hough Line detection is used in order to extract and use the straight information from the line elements, including the desired length, angle and area.

Extraction of points includes searching for all the intersection points of the straight elements extracted by the Hough Line detection, designating of the intersection points as the potential points and marking of such points on the Hough Line image. After then, all the quadrangles whose edges are the detected points are detected and among such quadrangles, the most ideal quadrangle in terms of size and proportion is designated as the central quadrangle. Based on the designated central quadrangle, the coordinates are extended to the entire grid pattern and the potential points are filtered. Then, misaligned coordinates are interpolated by way of drawing virtual straight lines and adjusting points to the straight lines. Interpolation originally means that lost information is filled with estimates but in this paper, interpolation is made by giving specific conditions, instead of estimates.

If corners of the pattern are detected properly, virtual straight lines are additionally drawn so as to select scope of view on the outside of the grid pattern. The virtual straight lines drawn on the outside of the grid pattern should be drawn in the place reduced at a rate of reduction of the distance between virtual straight lines drawn on the inside of the grid pattern. Moreover, reflecting the driver's desired scope of view and allowable value of quality of image synthesis, virtual straight lines are added at least 1 cell up to 5 cells. The actual length of 1 cell of the pattern here in is converted to $30 \mathrm{~cm}$. 


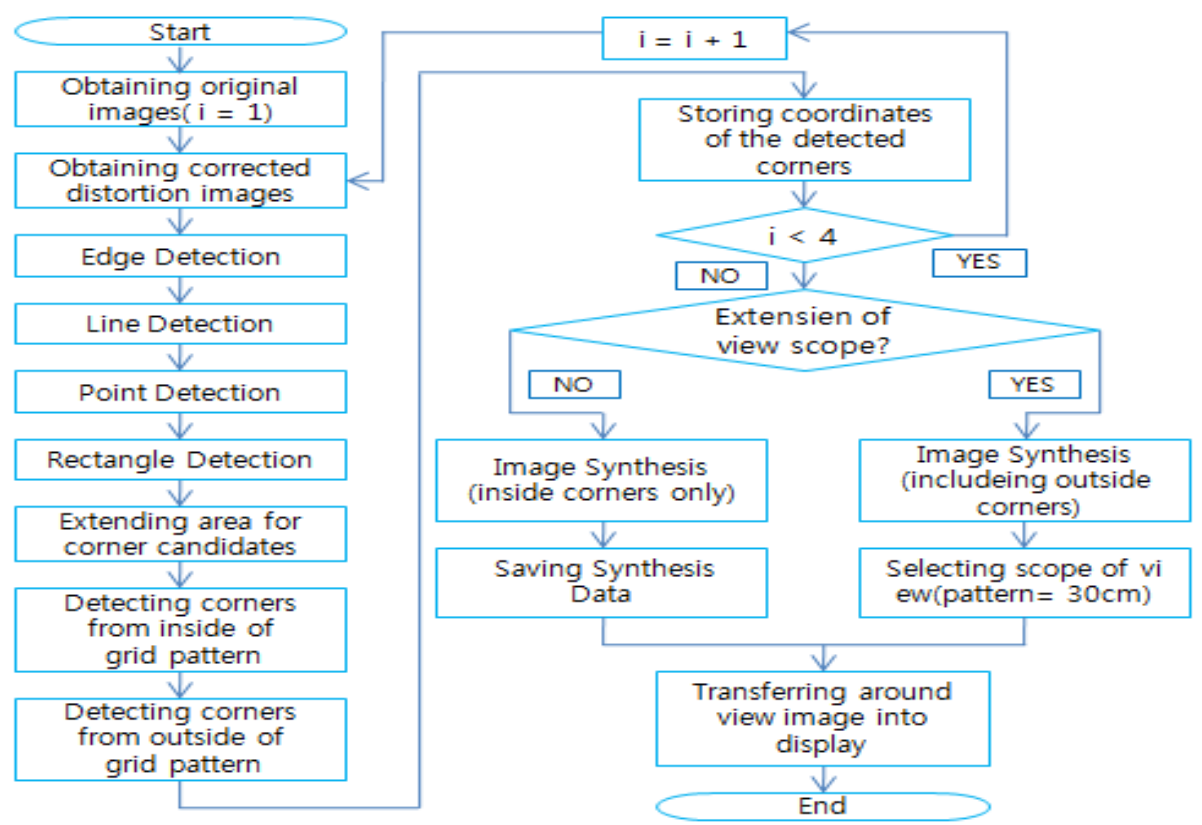

Figure 3. Adjusting Scope of Around View

Figure 3 shows an algorithm of inputting original images, detecting and synthesizing the external corners of the grid pattern. The implementation includes the methods to detect precise corners and select scope of view, while maintaining the existing algorithm frame of an AVM system.

\section{Implementation of an AVM System Selecting Scope of View}

In order to detect straight lines and corners, corrected distortion images should be used. It is because the basic image input by a camera has radial distortion due to distortion of the camera lens, as shown in Figure 4(a). By correcting such distortion, a corrected distortion image can be obtained as in Figure 4(b). By use of the corrected distortion image, straight lines and corners are detected.

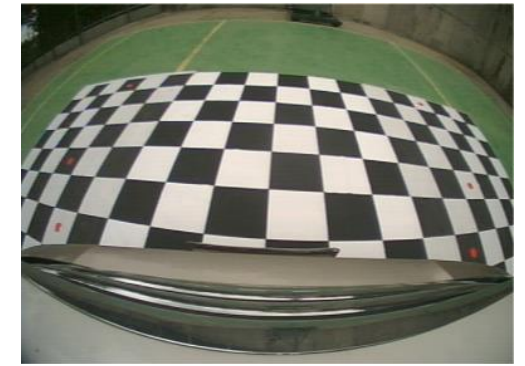

(a) Distorted Image

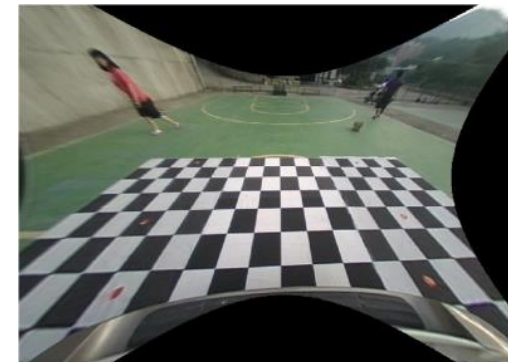

(b) Calibrated Image

\section{Figure 4. It Transform from (a) a Radial Distorted Image to (b) a Calibrated Image}

First of all, it is necessary to detect edges from the corrected distortion image. The edges herein refer to the border lines where the intensity of the image sharply changes. Figure 5(a) shows an image resulting from extraction of the edges from the corrected distortion image. After extracting edges from the corrected distortion image, all the straight lines on the inside of the image should be detected. For this, Hough Line detection is used. In the foregoing edge-extracted image, all the straight lines are detected by use of the Hough transformation, as shown in Figure 5(b). This paper implements a 
method to detect only the straight lines satisfying the desired elements by use of the Noise Filter.

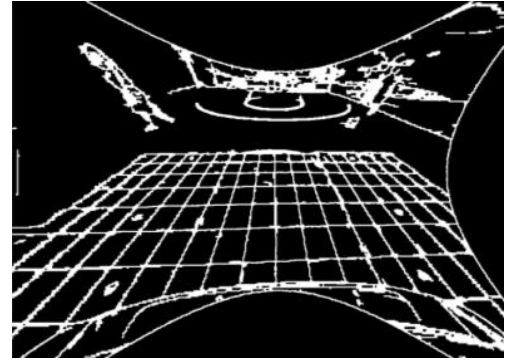

(a) Image which Extracts Edge

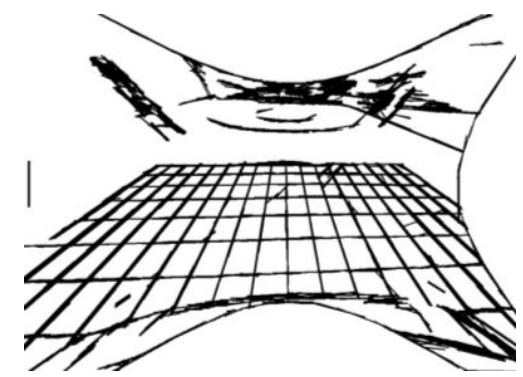

(b) Image which Extracts Straight Lines

\section{Figure 5. Process for Extracting Straight Lines}

Next, there is the implementation of a course of extracting potential areas, before detecting the corners of the grid pattern, by marking dots on the intersection points where straight elements meet on the image where straight lines are detected. As shown in Figure 6(a), other areas including the corners of the pattern were also extracted as potential areas.

In order to detect the center area, as shown in Figure 6(b) all the quadrangles which can be potential areas are made and among them, the quadrangles meeting the requirement for the central quadrangle are searched and indicated in color. The requirement for the central quadrangle refers to all the quadrangles whose horizontal to vertical ratio is appropriate. Among all the quadrangles detected as potential areas, the quadrangles close to the requirement for the central quadrangle are all marked.

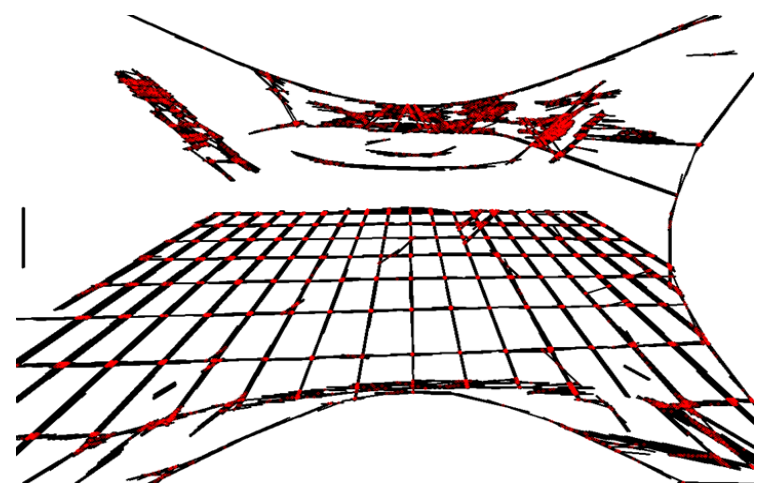

(a) Extracting Candidates for Corners

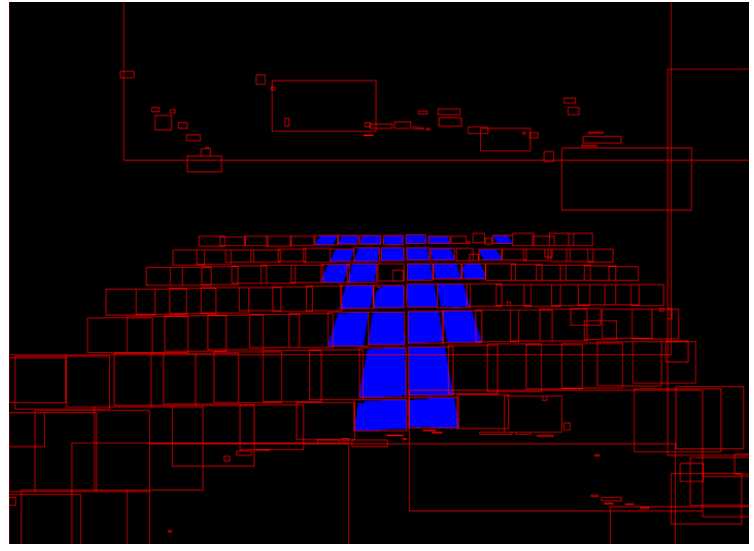

(b) Extracting Candidates for a Central Rectangle

Figure 6. Process for Extracting a Central Rectangle 
The potential central quadrangles are filtered once again and the most ideal central quadrangle is selected. In this filtering, the size of quadrangle is used to select the quadrangle of the size which is most similar to that of the real image. As a result, the most appropriate central quadrangle is detected as shown in Figure 7(a). Based on this, it is now necessary to detect the straight lines and corners of the grid pattern, respectively. Based on the coordinates of the central quadrangle, the potential area is extended to the entire grid pattern as shown in Figure 7(b), and the unnecessary dots from the selected potential areas are removed and the necessary areas are marked by dots.

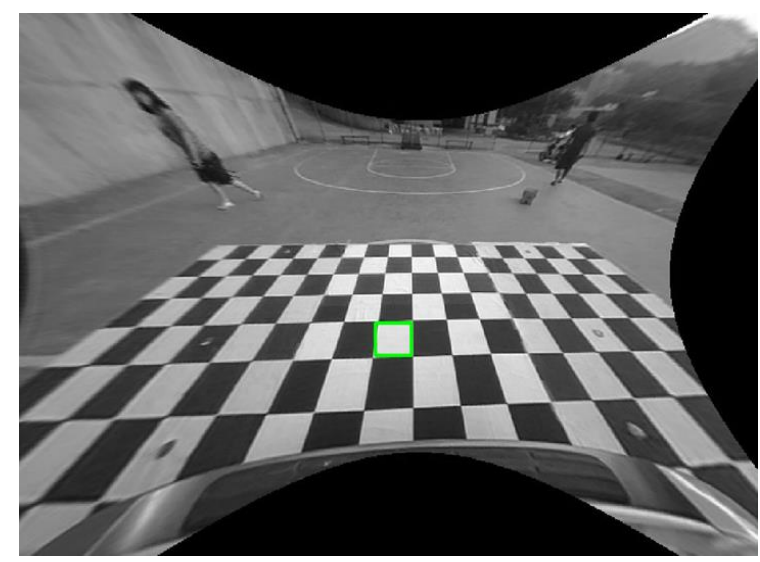

(a) Extracting a Best Profitable Central Rectangle

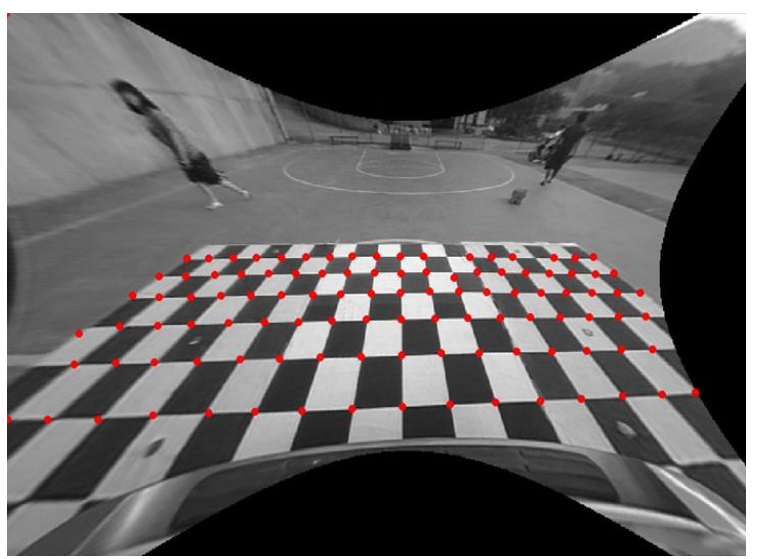

(b) Extending Candidates for Whole Grid Pattern

\section{Figure 7. Process which Extends Candidate Rectangles}

Next, by drawing the virtual straight lines which connect the corners of the grid pattern, whether the respective straight lines are identical to the corners of the grid pattern is examined. As Figure 8(a) shows, the corners of the grid pattern are almost identical to the intersection points of the straight lines. After then, as in Figure 8(b), virtual vertical lines are drawn on the outside of the grid pattern to obtain the coordinates of the corners so that a driver may select a wider scope of view. 


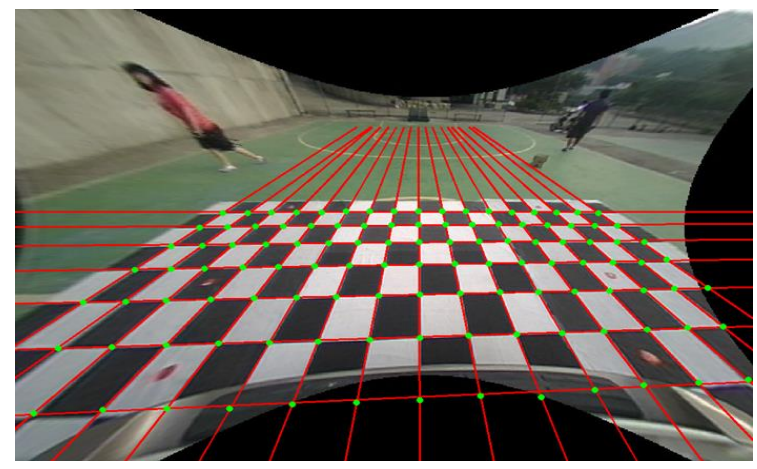

(a) Detecting Inner Corners

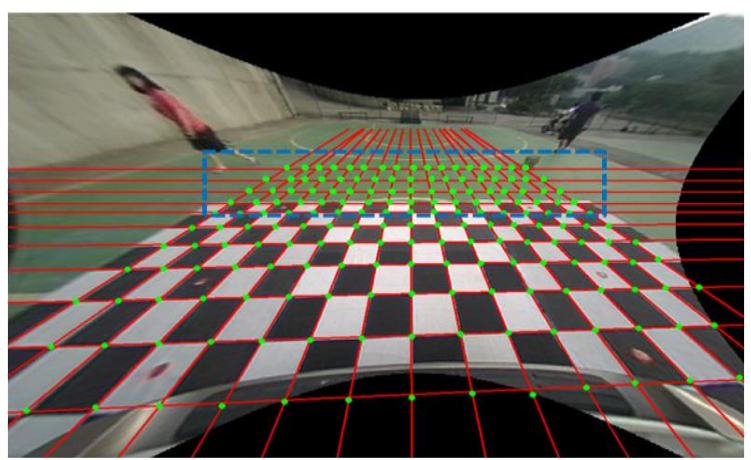

(b) Detecting Outer Corners

Figure 8. Detecting Corners from Inside/outside of Grid Pattern

If all the internal and external corners of the grid pattern are detected in regard to the four images from the front, rear, left and right sides, an around view image is synthesized by use of the respective corner coordinates. Figure 9(a) shows that the internal corner coordinates of the grid pattern are used for synthesization and Figure 9(b) shows that the entire internal and external corner coordinates of the grid pattern are used for synthesization. The quality of the image synthesis of the around view image using only internal corner coordinates of the grid pattern is better. However, despite lower quality, the around view image using all the internal and external corner coordinates of the grid pattern enables wider scope of view and a driver to select scope of view on his own.

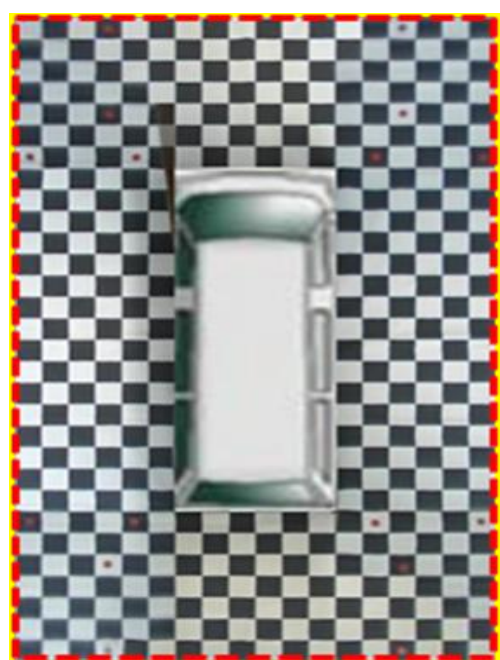

(a) Case of Inside Corners Only

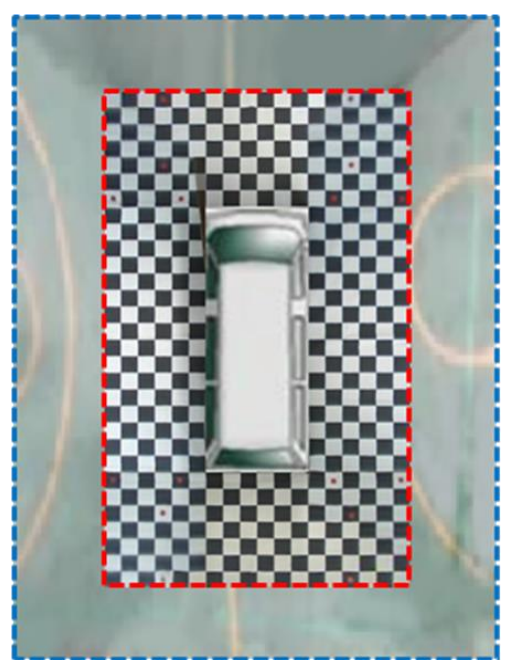

(b) Case of Including Outside Corners

Figure 9. Result of Image Synthesis 


\section{Conclusion}

This paper implements the methods to automatically detect straight lines and corners and to obtain an around view image by selecting the scope of view in an AVM system. If applying the AVM system proposed by this paper, the degree of automation is more advanced than that of the existing AVM system and thus, images can be synthesized effectively by use of the coordinates of the detected corners. Moreover, it has another strong point that a driver may select and identify the scope of view he wants through an around view image, instead of a fixed scope of view provided by the existing AVM system.

However, there is a weak point if the external coordinates of the grid pattern are synthesized: at the time of image synthesis from 5 or more cells, some parts are misaligned.

It will be necessary to improve the AVM system in the future, including increases in the quality of synthesis so as to provide wider scope of view for drivers, and design of an effective UI for a driver to easily set up his/her desired scope of view.

\section{Acknowledgements}

This work was supported by the Brain Busan 21 Project in 2014 and Dong-eui University Grant (2014AA486).

\section{References}

[1] A. Takahashi, Y. Ninomiya, M. Ohta, M. Nishida and M. Takayama, "Rear view lane detection by wide angle camera", Proc., IEEE Intelligent Vehicle Symposium, (2002). pp. 148-153.

[2] S. H. Cheon, Y. H. Yu and S. W. Jang, "An Efficient Camera Calibration Method in Embedded System Environment", Journal of the Korea Institute of Information and Communication Engineering, vol. 15, no. 1, (2011), pp. 623-626.

[3] Nissan's Elgrand Sensor System Simulates Birds-Eye View, http://www.newlaunches.com /archives/nissans_elgrand_sensor_system_simulates_birdseye_view.php.

[4] Y. Liu, K. Lin and Y. Chen, "Bird's-eye view vision system for vehicle surrounding monitoring", Proc., the 2nd international conference on Robot vision (RobVis'08). LNCS, Springer, pp. 207-218.

[5] G. Vass and T. Perlaki, "Applying and removing lens distortion in post-production", Second Hungrian Conference on Computer Graphics and Geometry, (2003).

[6] J. D. Kim and T. Y. Moon, "A Method ofr Improving Accuracy of Image Matching Algorithm for Car Navigation System", Journal of information and communication convergence engineering, vol. 9, no. 4, (2011), pp. 447-451.

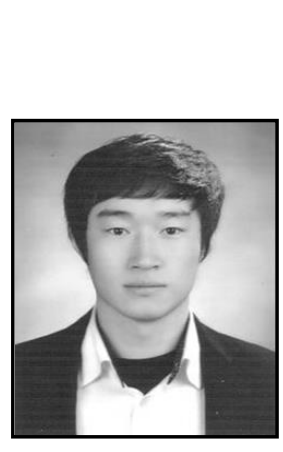

\section{Authors}

Sweung-Hwan Cheon received his B.S Degree from Department of Computer Science, Dong-eui University, Busan, Korea. He is studying as a candidate for M.S of Department of IT Convergence, Dong-eui University. His research interests include image processing and AVM systems.

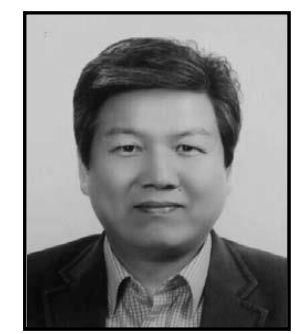

Jong-Wook Jang is a professor in the Computer Engineering Department at Dong-eui University. He received Ph.D in Computer Engineering in Pusan National University, Busan, Korea. His research interests are wired and wireless communication systems and Automotive Network. He is a member of the Korea Institute of Information and Communication Engineering(KIICE). 


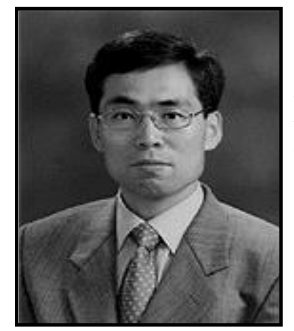

Si-Woong Jang received his M.S and Ph.D. degrees in 1993 and 1996, respectively from Department of Computer Science, Pusan National University. Busan, Korea. He is currently a professor with the Department of Computer Science, Dong-eui University, Busan, Korea. He worked for at research center of Daewoo Telecom from 1986 to 1993 . His research interests include vehicle network, AVM systems and database. 
International Journal of Control and Automation Vol. 7, No. 11 (2014) 